\title{
Maxillofacial Radiology 195
}

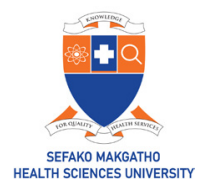

SADJ November 2021, Vol. 76 No.10 p644 - p645

N Nzima Chiloane ${ }^{1}$, S Rajbaran Singh ${ }^{2}$

ODONTOGENIC MYXOMA OF THE MAXILLA

A 48 year old asymptomatic male patient presented with a mass on the left maxilla with a reported awareness of two years. Clinical examination revealed normal mucosa overlying buccal and palatal swellings in dental region extending from the 23 to 27 . Tooth 26 was missing and teeth 24, 27 and 28 demonstrated displacement.
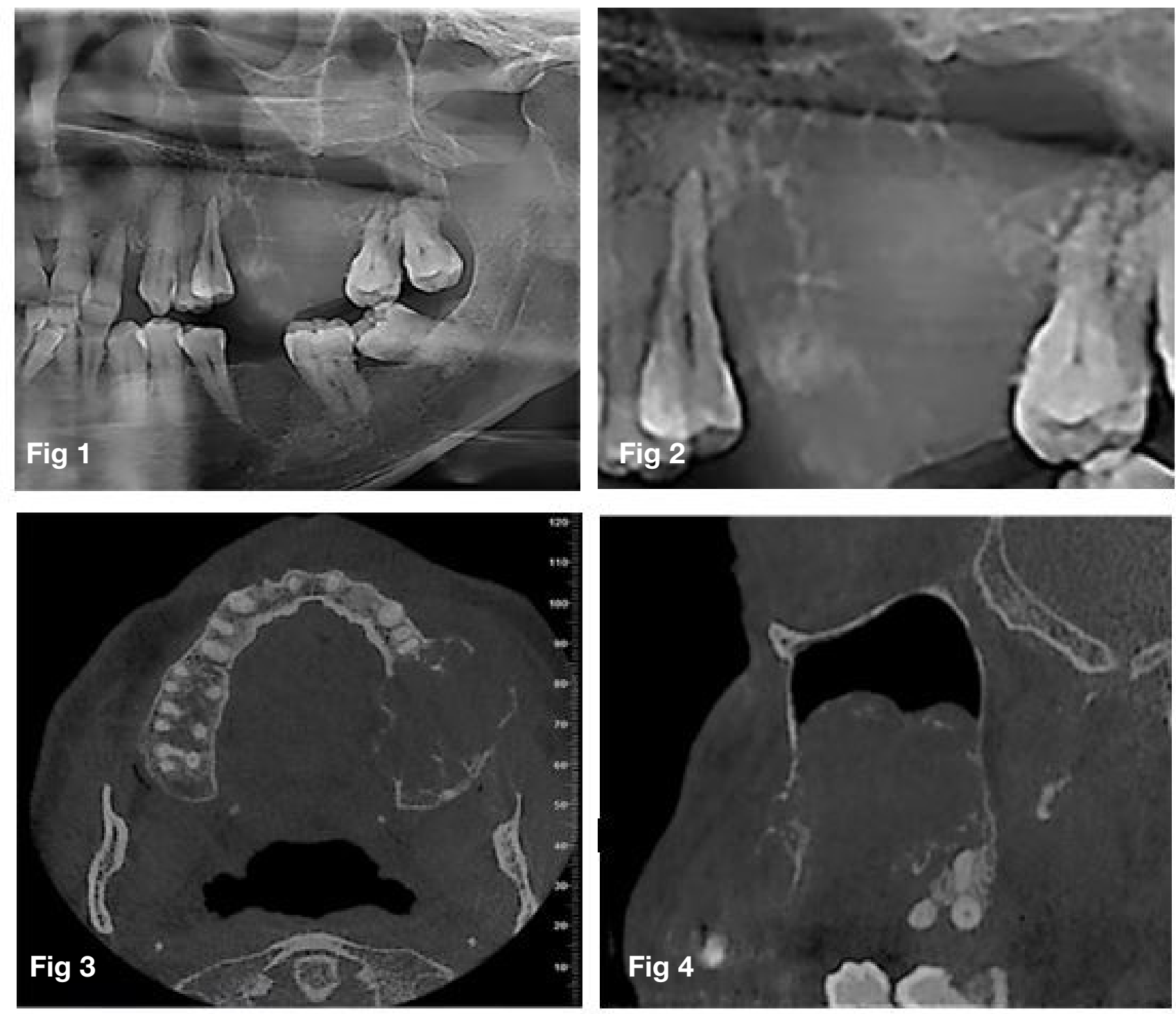

Author affiliations:

1. Nonhlanhla Nzima Chiloane: MDS, BDS, Fellowship, Health Sciences Education, School of Oral Health Sciences, Sefako Makgatho Health Sciences University, ORCID Number: 00000002-8200-1351

2. Sandeepa Rajbaran Singh: MSc, Dip Odont, BChD, Dip HRM, School of Oral Health Sciences, Sefako Makgatho Health Sciences University, ORCID number: 0000-0003-0658-2542

\section{Corresponding author:}

Nonhlanhla Nzima Chiloane: MDS, BDS, Fellowship, Health Sciences Education, Department of Maxillofacial and Oral Radiology, School of Oral Health Sciences, Sefako Makgatho Health Sciences University. Email: Nonhlanhla.nzima@smu.ac.za Cell: 0825678363
A panoramic radiograph (Figure 1) demonstrated an ill-defined radiolucent lesion of the posterior maxilla extending from tooth 25 to tooth 28, with a missing tooth 26 . Within the lesion, thin wispy radiopaque septae converging at right angles imparted a "tennis-racket" multilocular appearance (Figure 2). A radiographic impression of an odontogenic myxoma prompted further Cone Beam Computed Tomography (CBCT) scan. Axial views (Figure 3) demonstrated an expansive lesion causing thinning of cortical plates with focal areas of perforation. Sagittal views (Figure 4) demonstrated destruction of the sinus floor with infiltration of the inferior aspect of the maxillary sinus by a lobulated mass within which there are sparse trabeculae. 
Based on clinical presentation and radiographic appearance diagnoses of the lesion differentially included odontogenic myxoma, ameloblastoma and calcifying odontogenic cyst. Histopathological interpretation confirmed the lesion to be an odontogenic myxoma.

Odontogenic myxomas are benign, slow growing and locally aggressive odontogenic neoplasms bearing histological similarity to pulpal ectomesenchyme., ${ }^{1,2,3}$ With higher relative frequencies reported regionally in Asia, Europe and America, the global prevalence of odontogenic myxoma varies between 0.04 and $3.7 \% .^{2}$ Since initial description, several studies have reported variable radiographic features that overlap with benign and some malignant lesions. ${ }^{4}$ Radiographically, lesions appear as radiolucent or of mixed density. Lesional borders may be corticated, non-corticated, poorly defined, or diffuse. Larger lesions have been reported as multilocular while smaller lesions are reported as being unilocular. Arrangement of septae of residual bone within the lesion imparts patterning that has been described as honeycomb, soap bubble-like or resembling a tennis racket.5,6,7 The locally invasive nature of odontogenic myxoma is attributed to the expression of proteinases causing enzymatic degradation of extracellular matrix. This destruction is evident in the posterior maxilla when lesions demonstrate invasion of the maxillary sinus or the perforation of bone. The spectrum of clinical and radiological presentations justifies the timely review of this entity to enhance diagnosis, treatment planning and overall patient prognosis.

\section{References}

1. Martins H, Vieira E, Gondim A, Osório-Júnior H, da Silva J, da Silveira É. Odontogenic Myxoma: Follow-Up of 13 cases after conservative surgical treatment and review of the literature. J Clin Exp Dent. 2021;e637-41.

2. Hosalkar RM, Patel S, Pathak J, Swain N. Odontogenic Myxoma of Maxilla. J Contemp Dent. 2015 April; 5(1):27-30.

3. Kheir E, Stephen L, Nortje C, Janse van Rensburg L, Titinchi $F$. The imaging characteristics of odontogenic myxoma and a comparison of three different imaging modalities. Oral Surg Oral Med Oral Pathol Oral Radiol. 2013 Oct;116(4):492-502.

4. Shivashankara C, Nidoni M, Patil S, Shashikala KT. Odontogenic myxoma: a review with report of an uncommon case with recurrence in the mandible of a teenage male. The Saudi dental journal. 2017Jul 1;29(3):93-101.

5. Chuchurru JA, Luberti R, Cornicelli JC, Dominguez FV. Myxoma of the mandible with unusualradiographic appearance. Journal of oral and maxillofacial surgery. 1985 Dec 1;43(12):987-90.

6. Cuestas-Carnero R, Bachur RO, Gendelman H. Odontogenic myxoma: report of a case. Journal of oral and maxillofacial surgery. 1988 Aug 1;46(8):705-9.

7. MacDonald-Jankowskia DS. Yeung RWK, Li T, Leeb KM . Computed tomography of odontogenic myxoma. Clinical Radiology.2004: 59, 281-287

\section{Do the CPD questionnaire on page 649}

The Continuous Professional Development (CPD) section provides for twenty general questions and five ethics questions. The section provides members with a valuable source of CPD points whilst also achieving the objective of CPD, to assure continuing education. The importance of continuing professional development should not be underestimated, it is a career-long obligation for practicing professionals.

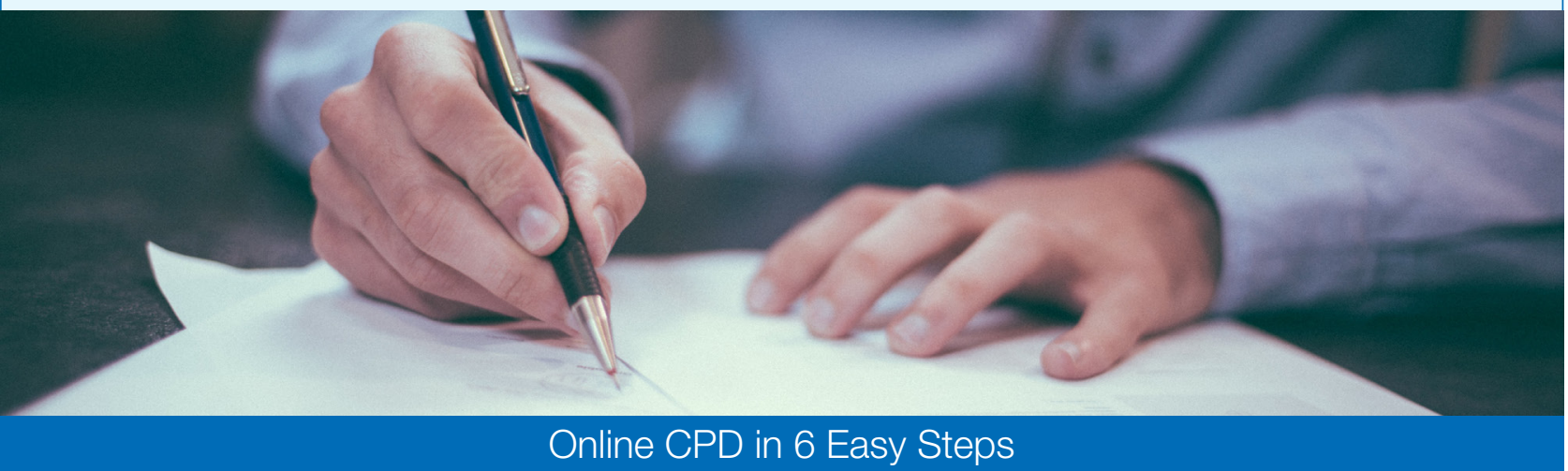

Go to the SADA website www.sada.co.za

2 Log into the 'member only' section with your unique SADA username and password.

3 Select the CPD navigation tab.

4 Select the questionnaire that you wish to complete.

5 Enter your multiple choice answers. Please note that you have two attempts to obtain at least $70 \%$.

$6 \quad$ View and print your CPD certificate. 